\title{
WORKING MEMORY IN PRIMATE SENSORY SYSTEMS
}

\author{
Tatiana Pasternak ${ }^{\star}$ and Mark W. Greenlee \\ Abstract | Sensory working memory consists of the short-term storage of sensory stimuli to guide \\ behaviour. There is increasing evidence that elemental sensory dimensions - such as object \\ motion in the visual system or the frequency of a sound in the auditory system - are stored by \\ segregated feature-selective systems that include not only the prefrontal and parietal cortex, but also \\ areas of sensory cortex that carry out relatively early stages of processing. These circuits seem to \\ have a dual function: precise sensory encoding and short-term storage of this information. New \\ results provide insights into how activity in these circuits represents the remembered sensory stimuli.
}

${ }^{*}$ Departments of Neurobiology \& Anatomy, Brain \& Cognitive Science and Center for Visual Science, Box 603, University of Rochester, Rochester, New York 14642, USA. ${ }^{\ddagger}$ Institute for Experimental Psychology, University of Regensburg, D-93050 Regensburg, Germany. Correspondence to T.P. e-mail:

tania@cvs.rochester.edu doi:10.1038/nrn1603

Published online 14 January 2005
Perception and memory are often depicted as separate processes, and researchers have often characterized the brain circuits that underlie perception and memory as distinct (and often anatomically distant) from each other. This review focuses on the neurocognitive mechanisms of working memory in primate sensory systems — the brief storage of sensory information that is used to guide our ongoing actions ${ }^{1}$ — and its relationship to the mechanisms that underlie the processing of this information. We concentrate on studies that require subjects to hold information temporarily in short-term storage to perform a discrimination on a trial-by-trial basis. Various approaches have been used to investigate sensory working memory, ranging from psychophysics and functional imaging to single-cell recordings, electrical brain stimulation and studies of monkeys and humans with lesions. Psychophysical studies have focused on the properties of stimulus representations that are retained in working memory, whereas neurophysiological, imaging and lesion studies have focused on the regions in sensory cortex that analyse various stimulus features as well as focusing on the representation of sensory stimuli in regions that are traditionally associated with working memory, such as the prefrontal cortex (PFC) (BOX 1). The evidence that has emerged from these studies supports the idea that, in the primate brain, elemental sensory dimensions are represented by segregated memory systems that probably involve those cortical areas that are involved in encoding stimulus features, as well as prefrontal and parietal regions. This circuitry supports the brief retention of sensory information for use in sensory-guided behaviour. Although much of the information about sensory working memory comes from studies of the visual system, there is a growing body of literature that deals with the storage of information in other sensory modalities, particularly tactile and auditory. The goal of this review is to discuss the key evidence that sensory cortical areas are an active component of the circuitry that underlies shortterm retention of sensory signals. To facilitate comparison with work in non-human primates, this review is restricted to studies that used non-linguistic stimuli.

\section{Visual working memory}

Psychophysics. Visual working memory has been studied in subjects who performed tasks that involved delayed discrimination of basic stimulus attributes (such as size, orientation, contrast or direction of motion; FIG. 1a,b). The use of such stimuli offers the opportunity to test the ability to store parametric values of individual stimulus dimensions ${ }^{2}$, and provides a quantitative measure of sensory storage by determining the threshold values that are needed for reliable retention of a sensory stimulus. Because the neuronal mechanisms that are involved in the encoding of many of these attributes have been extensively studied ${ }^{3}$, we can specify the effects of such stimuli on the initial stages of visual processing and can better understand the mechanisms that underlie their subsequent storage. 


\section{Box 1 | Sensory signals in the prefrontal cortex}

The prefrontal cortex (PFC) consists of several cytoarchitectonically defined regions. There is anatomical and physiological evidence that the PFC is functionally segregated with respect to sensory modalities and stimulus features ${ }^{85-87}$. The physiological evidence includes reports that neurons in regions of the PFC that receive inputs from sensory neurons processing a particular stimulus feature respond selectively to that feature ${ }^{88-90}$. On the other hand, there is accumulating evidence that many PFC neurons integrate information across sensory modalities ${ }^{91,92}$ and across different stimulus attributes within the same modality ${ }^{93}$. One way to reconcile the apparent anatomical and functional segregation within the PFC with the presence of neurons that integrate information between different modalities and different stimulus features is to engage intrinsic connectivity within the $\mathrm{PFC}^{94}$.

It is widely accepted that the PFC has a key role in sensory working memory. Although the mechanisms that underlie sensory storage are not well understood, most models of working memory (BOX 2) include cortical areas that process sensory information in the underlying circuitry ${ }^{95,96}$. Indeed, many neurons in the PFC respond to the sensory stimuli that are used in working memory tasks ${ }^{63,88,97}$. Furthermore, during a memory delay after a sensory cue, many PFC neurons show elevated cue-related activity that reflects the attributes of the relevant sensory modality ${ }^{88,97-101}$. This activity has been interpreted as a mnemonic code that is used to retain sensory information (see, for example, REF. 102).

There is ample evidence for feedforward and feedback connections within sensory cortical areas and between those areas and the $\mathrm{PFC}^{103}$. Although the precise nature of the interactions between neuronal populations in the PFC and sensory cortex during working memory tasks is not well understood, there is evidence for a dynamic interplay between them. The prevailing view, which is supported by studies of inferotemporal (IT) cortex in the absence of inputs from the PFC, is that the PFC exerts 'top-down' influences on sensory cortex ${ }^{44,104-106}$. Additional support for top-down control comes from the observation that target selectivity seems to occur more rapidly in the PFC $^{99}$ than in IT cortex ${ }^{31,107}$.
Many studies have shown that fundamental stimulus features such as orientation, size, speed or direction of motion can be retained in memory for many seconds, with little loss of information (FIG. 1b). There is also evidence that these distinct stimulus dimensions are stored by separate, feature-selective mechanisms ${ }^{4}$. For example, different features can be retained in working memory for different lengths of time (FIG. 1b): spatial frequency $(\text { size })^{5-8}$, orientation ${ }^{9,10}$ or speed of motion can be retained for several seconds with little loss of precision, whereas the retention of luminance contrast ${ }^{11,12}$, texture ${ }^{13}$, small spatial offsets (vernier stimuli) ${ }^{14}$ and direction of motion ${ }^{15}$ seem to be less robust. This apparently selective decay of information could indicate that there are separate storage processes for different stimulus features ${ }^{16}$. However, because the procedures that are used to study the retention of various stimulus attributes were not matched, some of the differences in decay functions could partly be due to procedural differences.

More compelling evidence in support of featureselective retention mechanisms comes from studies that used interference consisting of an irrelevant stimulus, or 'memory mask', that is introduced during the memory delay (FIG. 2a). If such stimuli interfere with the memory representation, they produce a decrease in performance on the task. By manipulating the properties of the mask it is possible to gain insights into the nature of the remembered stimulus. For example, the ability of a masking grating to interfere with the delayed discrimination of spatial frequencies is greatest when the spatial frequency of the mask differs from that of the remembered stimulus by at least an octave ${ }^{5}$. The effect is specific to the spatial frequency of the remembered grating and is unaffected by changes in the orientation of the mask. This selective interference indicates that information about spatial frequency is preserved by specialized mechanisms that are closely associated with those involved in its encoding, and are distinct from mechanisms that are concerned with stimulus orientation.

To investigate the dynamics of the storage process, another study used a spatial frequency discrimination task and introduced an interfering stimulus at different points during a $10-\mathrm{s}$ delay ${ }^{17}$. The interfering stimulus was most effective when it was presented early in the delay, indicating that visual working memory might be a two-stage process: an initial short-lived perceptual encoding stage that is vulnerable to masking, and a more robust later stage. Memory tasks that involve visual motion are also more vulnerable to interference early in the delay period ${ }^{18}$.

Information about the speed and direction of visual motion can also be accurately preserved, sometimes for many seconds ${ }^{6,19}$. To examine the nature of the retained speed information, an intervening mask, also consisting of a moving stimulus, was used while subjects compared two stimuli that moved at different speeds. The mask reduced the accuracy of performance only if its speed did not match the speed of the remembered stimulus, irrespective of its direction of motion. The selectivity of the masking effect, which resembled that of masking effects in memory for spatial frequency ${ }^{5}$, indicates that speed is faithfully preserved in memory and that the mechanisms of this preservation are relatively narrowly tuned. It also shows that speed and direction might be stored by separate mechanisms.

Selective retention of information about motion direction has been shown in monkeys that were required to compare two random-dot moving stimuli separated by a delay ${ }^{15,20,21}$. The monkeys remembered the direction of coherent motion with only a modest decrease over time and reliably retained the direction of motion of stimuli with relatively low levels of coherence for as long as 4-6 s (REF. 15). In a subsequent study, the two comparison stimuli were spatially separated and a random-motion mask was introduced during the delay in either the sample location or the location of the upcoming test ${ }^{18}$ (FIG. 2a-c). The effect of the mask was specific to its properties and location (FIG. 2d,e). This selective interference indicates that the sensory attributes of visual motion are precisely preserved, which supports the idea that mechanisms that are involved in the processing of visual motion are also involved in its storage. Furthermore, this selectivity of masking effects resembles the selectivity that was seen in studies of memory for spatial frequency and speed ${ }^{5,6}$, indicating that these different memory networks might share a similar architecture.

Evidence that the storage of visual motion in these tasks is specific for the location of the stimulus comes from a study ${ }^{21}$ that investigated the effect of spatial separation between the sample and test stimuli. The authors found that if the two comparison stimuli were 
a

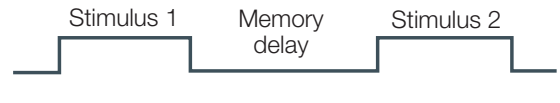

Vernier

Contrast

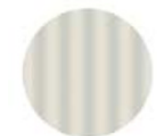

Orientation

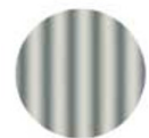

Spatial

frequency

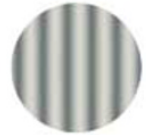

Speed

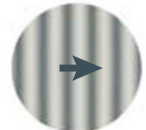

Direction

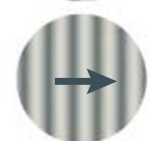

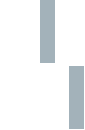
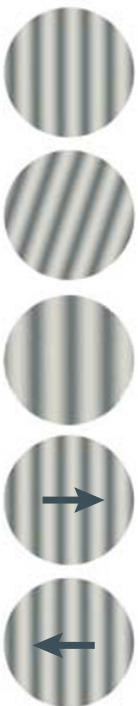

b

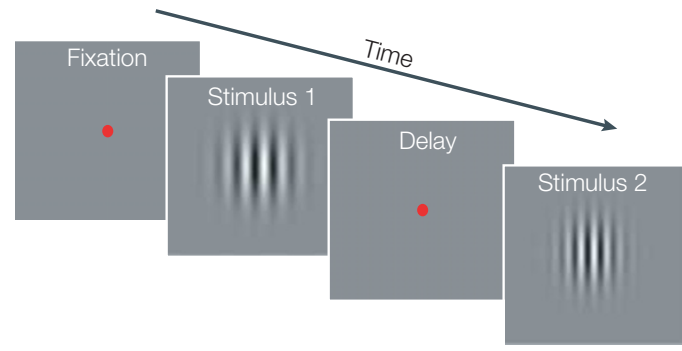

c

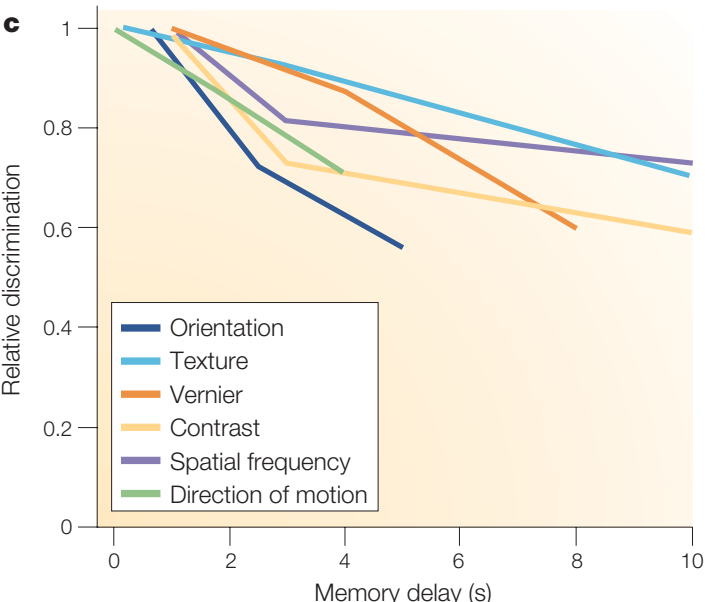

Figure 1 | Visual working memory for selected stimulus attributes. a | Delayed discrimination task and examples of visual stimuli that are commonly used to study the retention of stimulus attributes. Sinusoidal gratings are used to determine thresholds for discriminating contrast, orientation or other grating attributes. These thresholds (not shown) provide a measure of how well a given attribute is retained in memory. b | Typical behavioural paradigm. The trial begins with the onset of a central fixation point followed by a brief (200-500 ms) presentation of stimulus 1 (the 'sample' or 'cue'). During the delay, the fixation target is present to aid fixation. At the end of the delay, the subject views stimulus 2 (the 'test'), which differs from stimulus 1 along the dimension of interest (spatial frequency; for instance, bar width). The subject reports whether the second stimulus matches the first with respect to a given stimulus attribute. In the example shown, stimulus 2 has a higher spatial frequency than stimulus 1. c | Normalized discrimination sensitivity for various visual attributes plotted as a function of delay. The different curves were re-plotted from studies in which memory performance was evaluated for texture ${ }^{13}$, contrast ${ }^{7}$, vernier ${ }^{14}$, motion direction ${ }^{15}$, spatial frequency ${ }^{7}$ and orientation ${ }^{10}$. Although the retention of stimulus attributes degrades with time, this degradation is modest. Even after a 10-s delay, representation of some stimulus dimensions is degraded by no more than $25 \%$.

VENTRAL AND DORSAL VISUAL STREAMS

Visual information coming from $\mathrm{V} 1$ is processed in two interconnected but partly dissociable visual pathways, a 'ventral' pathway extending into the temporal lobe, which is thought to be primarily involved in visual object recognition, and a 'dorsal' pathway extending into the parietal lobes, which are thought to be more involved in extracting information about 'where' an object is or 'how' to execute visually guided action towards it. spatially separated by more than a 'critical' separation, the threshold for discrimination of motion was elevated. They used the size of this separation to estimate the spatial extent of the mechanisms that were involved in the performance of the task. The critical separation increased with retinal eccentricity, and its size coincided with the size of receptive fields in cortical area MT, an area that is strongly associated with processing of visual motion, indicating that MT might be involved in performing the task. This finding supports the idea that retinotopically organized visual areas are involved in the storage of motion information.

Sekuler and colleagues used a different approach to examine the nature of stimulus representation in memor $\mathrm{y}^{22,23}$. In their task, subjects were presented with two consecutive texture stimuli followed by a probe stimulus, each consisting of vertical and horizontal sinusoidal gratings of varying spatial frequencies. The subjects had to judge only the vertical component of these textures and report whether the probe stimulus contained the same spatial frequency as either of the two preceding stimuli. The results supported the idea that the remembered spatial frequency of each stimulus evaluated during the trial has a separate representation in memory that approximates the original stimulus but that is subject to noise and therefore constitutes a 'noisy exemplar'.

Although the mechanisms that underlie the processing of colour have been extensively studied ${ }^{24}$, relatively little is known about working memory for colour. Subjects retain information about hue with great precision ${ }^{25}$, indicating that regions of the brain that are concerned with sensory processing of colour are also involved in this form of working memory. There is also evidence that the colour of an object can be processed separately from its location. In one study, colour or location distractors were introduced during the delay between two stimuli that differed in either colour or location ${ }^{26}$. Location distractors interfered only with memory for location and not colour, whereas colour distractors interfered only with memory for colour, supporting the idea that memory for colour and spatial location are handled by separate systems. This conclusion is consistent with the notion that colour and location are processed by separate cortical visual pathways - the vENTRAL AND DORSAL STREAMS ${ }^{27}$ and indicates that this separation also holds for the temporary storage of these signals. 
a

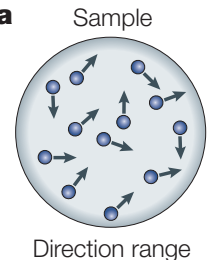
$180^{\circ}$

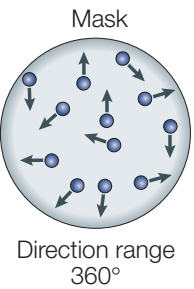

$360^{\circ}$

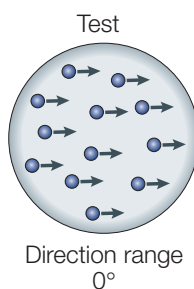

$0^{\circ}$

b Mask in sample location
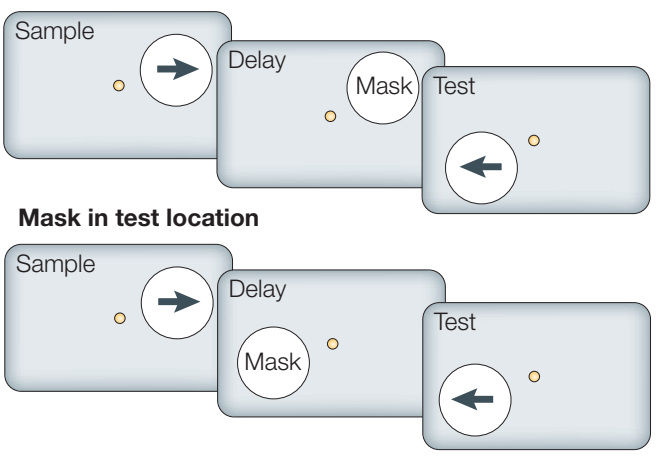

c

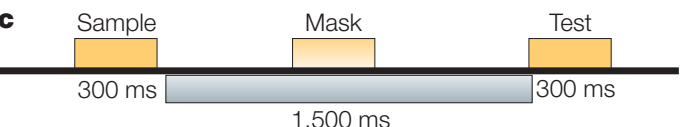

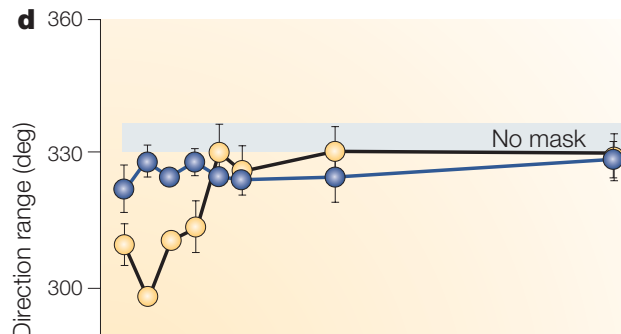
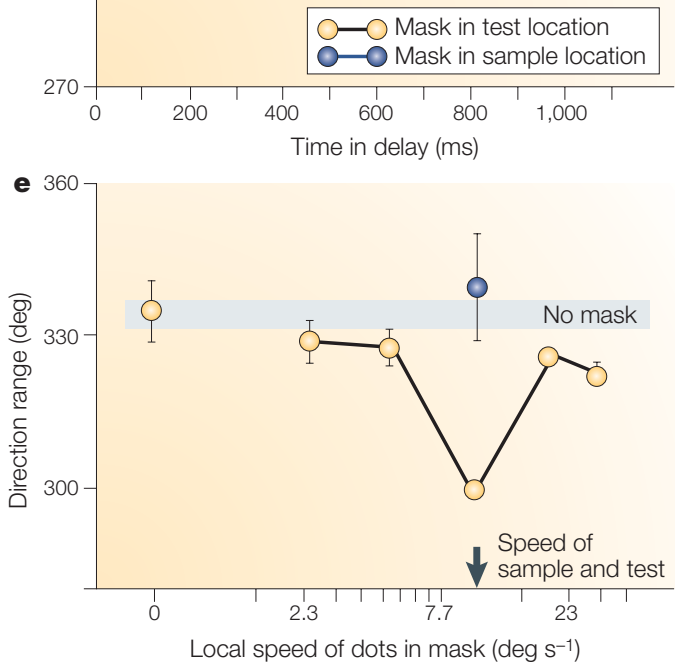

Figure 2 | Visual working memory for stimulus direction. a | Stimuli used to measure retention of motion direction in monkeys. b | Behavioural task. On each trial, the monkeys compared two directions of motion, sample and test, which were separated by a delay and in space. The sample consisted of random dots that moved in a variable range of directions; in the test stimulus, all the dots moved in the same direction. The monkeys reported whether the sample and test had the same mean direction of movement. The mask, consisting of dots moving in random directions ( $360^{\circ}$ range), was introduced during the delay at the location of the preceding sample (upper row) or the upcoming test (lower row). c | Temporal parameters of the task. d | Temporal and spatial specificity of the remembered stimulus. Direction range thresholds are plotted as a function of the mask onset and location. Blue circles indicate thresholds measured with the mask at the sample location; yellow circles indicate thresholds measured with the mask at the test location. Grey bar, threshold value $( \pm$ SEM) without the mask. The mask interfered with performance only when presented early in the delay at the location of the upcoming test. This indicates that the representation of remembered direction is spatially localized, is transferred to the site of the anticipated test, and is most vulnerable early in the delay. Deg, degrees. e Stimulus specificity of the remembered sample. The local speeds of dots in the mask were varied. The mask decreased performance only when its local speeds matched the speed of the remembered sample, even though the monkey was not instructed to remember stimulus speed. This demonstrates that information about speed is preserved along with information about stimulus direction. Modified, with permission, from REF. 18 @ (2002) Elsevier Science.

Overall, the mechanisms that preserve basic attributes of visual stimuli can be characterized as narrowly tuned, spatially localized filters, thereby supporting a model of working memory that involves the contribution of sensory cortical areas.

Physiology. Only a handful of neurophysiological studies have directly investigated the involvement of visual cortical areas in working memory. Until recently, much of the neurophysiological work on visual working memory has focused on the inferotemporal (IT) cortex, a relatively advanced stage of the ventral visual stream that is important for processing complex shapes ${ }^{27}$. This work has shown that IT neurons in monkeys that have been trained to remember objects are active during the delay period after they have been shown the object, and that this activity is related to the remembered colour or shape ${ }^{28-31}$. Memory-related activity elicited by both colour and motion has also been recorded in area V4, an earlier component of the ventral visual stream ${ }^{32,33}$.
Recordings from regions in the parietal cortex that represent more advanced stages of processing in the dorsal visual stream also showed memory-related activity. For example, neurons in area $7 \mathrm{a}$ and the lateral intraparietal cortex (area LIP) fire while monkeys remember spatial locations ${ }^{34}$. Ferrera et al. ${ }^{32}$ also found memory-related modulation of activity in area 7a while monkeys retained information about motion direction. These effects were largely absent in area MT, an earlier component of the dorsal pathway that is specialized for processing visual motion.

However, a recent study provided evidence for memory-related activity in area $\mathrm{MT}^{35}$. In that study, the activity of MT neurons was recorded during a 1.5-s delay in a motion discrimination task (FIG. 3a). A typical neuron (FIG. 3a) was active not only in response to the sample and test stimuli, but also during the delay, when it showed a characteristic pattern of activity that consisted of a small burst of firing early in the delay, subsequent suppression of firing and then reactivation just before the presentation 


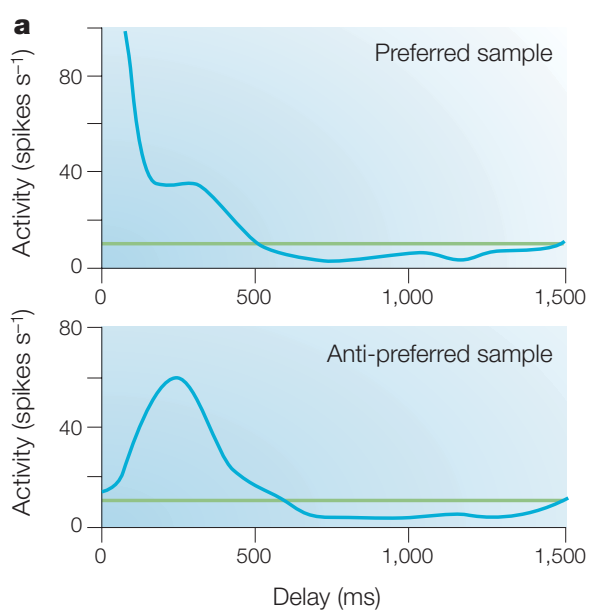

C

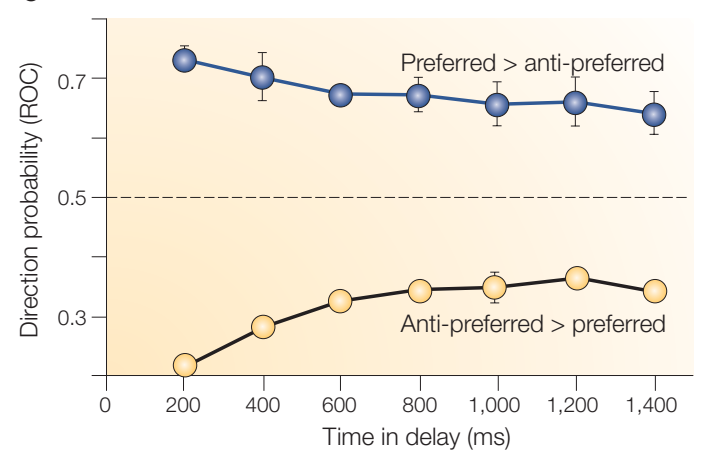

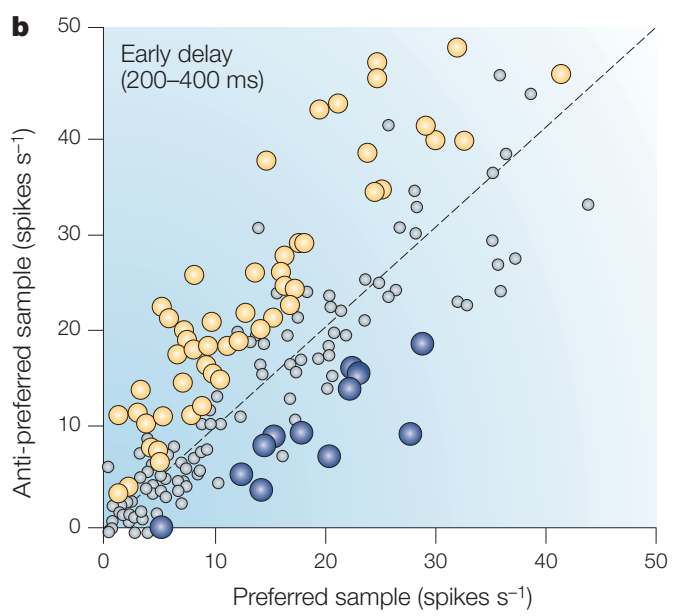

d

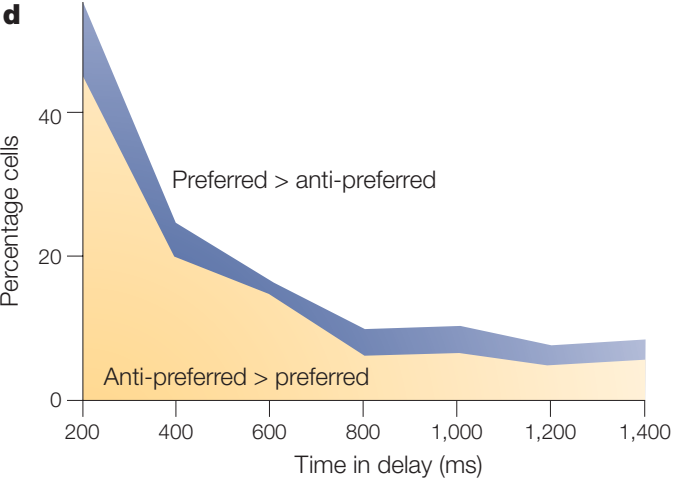

Figure 3 | Directional signals in area MT during the memory delay. a | Delay activity of an MT neuron during the working memory for motion task. Average firing rates recorded during the delay in a single testing session are shown as spike density functions for trials with sample moving in the preferred (top plot) and anti-preferred direction (bottom plot). Baseline activity (horizontal line) was recorded during fixation. This neuron shows a typical pattern of delay activity: brief early activation, prolonged suppression followed by a gradual increase in firing in anticipation of the end of the delay. $\mathbf{b} \mid$ Firing rates of individual neurons during early delay (200-400 ms) after a sample moving in the preferred and the anti-preferred direction. Although the firing rates of many neurons after exposure to stimuli moving in the two directions were not significantly different (grey circles), there was a large group of cells with activity that depended on the direction of the preceding sample (yellow and blue circles; $t$-test $p>0.05$ ). c $\mid$ Receiver operating characteristic (ROC) analysis of firing rates recorded during the delay. The delay was divided into 7 independent 200-ms periods. Values significantly different from 0.5 indicate firing rates that carry directional signals ${ }^{35}$. Two populations of cells that signal sample direction were identified: cells with higher firing rates after the preferred direction (blue) and cells with higher rates after the anti-preferred direction (yellow). $\mathbf{d} \mid$ The proportion of neurons contributing to the curves shown in $\mathbf{c}$. More cells showed a significant ROC score after the anti-preferred sample. Data from REF. 35 @ (2000) American Physiological Society.

of the test stimulus. Analysis of the activity that was recorded during the delay revealed significant differences in firing rates with respect to the direction of the preceding sample. Early in the delay, about $300 \mathrm{~ms}$ after sample offset, a large proportion of neurons had significantly higher firing rates after a stimulus that moved in the antipreferred direction (yellow circles; FIG. 3b) and a smaller proportion fired significantly more after a stimulus that moved in the preferred direction (blue circles). ROC (receiver operating characteristic) analysis of firing rates recorded during this period and during the rest of the delay revealed the presence of reliable directional signals: most neurons fired more after the preferred direction, whereas a small proportion of neurons fired more after the anti-preferred direction (FIG. 3c,d). So, throughout the delay, the direction of the remembered stimulus was represented in area MT, consistent with the idea that this sensory region of cortex participates in the retention of visual motion information ${ }^{15,20}$.
Imaging. The use of functional imaging to study the neural correlates of perceptual memory has been discussed in previous reviews ${ }^{36,37}$. Here, we highlight studies that focus on the involvement of sensory corti$\mathrm{cal}$ areas in visual working memory. These studies also support the view that sensory cortical areas participate in working memory, although the results seem to depend on the type of working memory task that is used. For example, imaging studies show greater memory-related activity in sensory cortex during delayed discrimination tasks than during ${ }_{N \text {-BACK TASKS. During a }}$ delayed discrimination task that involved spatial frequencies, there was a delay-related increase in activity not only in the prefrontal and posterior parietal cortex but also in the occipital cortex, which processes visual information ${ }^{38}$. Similarly, delay-related increases in visual cortical activation occurred during delayed discrimination of stimulus orientation $s^{39}$. However, studies that used $n$-back tasks reported strong activation 

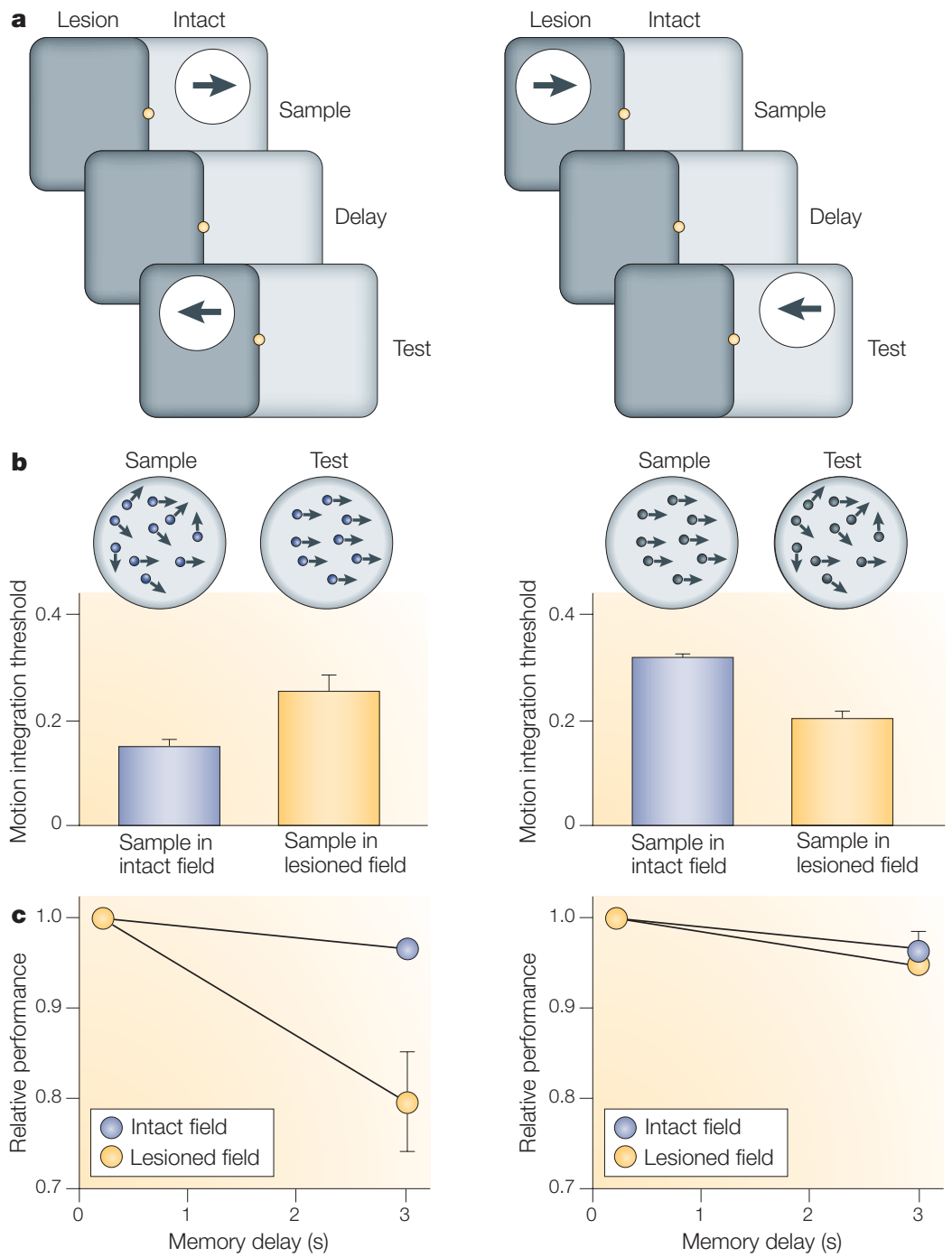

Figure 4 | Effect of MT/MST lesions on discrimination and memory of the direction of complex motion. a | Monkeys with unilateral MT/MST lesions performed the task with sample and test stimuli in opposite hemifields so that one was placed in the lesioned hemifield and the other in the corresponding location in the intact hemifield. b | Normalized direction range thresholds ((360range threshold/360) measured when the delay between sample and test was minimal (0.2 s). The sample or the test stimulus was composed of dots moving in a range of directions (blue), whereas the other contained only coherent motion. 'Sample in intact field' (blue) indicates that the sample was presented in the intact visual field and that the test was presented in the lesioned hemifield. 'Sample in lesioned field' (yellow) indicates that the sample was presented in the lesioned hemifield and that the test was presented in the intact hemifield. Thresholds were poorer whenever the stimulus containing non-coherent motion was placed in the lesioned field. $\mathbf{c} \mid$ Effect of delay on performance for two direction range tasks. Thresholds were measured with both stimuli in the intact (blue circle) or lesioned (yellow circle) hemifield. Range thresholds were measured either by varying the range of directions in the sample while the test moved coherently (left graph) or by varying the range of directions in the test while the sample moved coherently (right graph). Thresholds were normalized to the data measured at 0.2-s delay. Error bars show SEM. A delay-specific deficit was present only when the remembered stimulus (sample) contained a broad range of directions and required integration. Modified, with permission, from REF. 15 ○ (2000) Oxford University Press.

mainly in regions outside sensory cortex, such as the prefrontal and posterior parietal cortex, with little selective activation in visual cortex ${ }^{36,40-42}$. One possible explanation for the absence of delay activation in visual cortex in $n$-back tasks is that memory representation of target and non-target stimuli used in such tasks interact, and this interaction might impede on-going cortical activity.
Lesions. One of the first studies to investigate the effects of sensory cortical lesions on working memory was performed by Fuster and colleagues, who applied local cooling to IT cortex in monkeys during a DELAYED MATCHTO-SAMPLE task ${ }^{43}$. Cooling produced severe impairments in performance, but only when there was a long memory delay between the sample and test stimuli. In a subsequent study ${ }^{44}$, Fuster et al. examined the effects of cooling of the dorsolateral PFC on the performance of the same task and on the activity of neurons in IT. Cooling affected task performance and altered the stimulus selectivity of neuronal activity in IT during the delay period. These results show that interactions between prefrontal and IT neurons probably have an important role in working memory. The role of IT cortex in retaining information about visual objects or colour has been confirmed by other lesion studies ${ }^{45,46}$, providing physiological evidence for the involvement of IT cortex in visual working memory.

As described above, neurons in area MT carry signals about the remembered direction during a memory-formotion task ${ }^{35}$. The involvement of area MT in working memory has also been documented in a lesion study ${ }^{15}$. The authors used the same memory task and investigated the effects of unilateral lesions of area MT and the neighbouring area MST (both areas of extrastriate cortex that are involved in processing visual movement). By placing either a sample stimulus or a test stimulus in the intact or lesioned visual hemifield, they could assess separately the contribution of this area to the encoding and storage of visual motion. When the sample stimulus consisted of random dots moving in a broad range of directions (complex motion), MT/MST lesions disrupted both encoding of these stimuli and their retention at a longer delay (FIG. 4). However, when a coherent sample (all dots moving in the same direction) was displayed in the lesioned visual hemifield, there was no deficit in perceptual thresholds and no additional deficit with a longer delay. This result showed that encoding and storage seem to be closely linked; when the lesion affected stimulus encoding, retention of that stimulus was also degraded.

Investigations in patients with focal brain damage also support the involvement of regions that process sensory information in visual working memory. The short-term retention of spatial frequency ${ }^{47}$, speed of motion $^{48}$, colour ${ }^{49,50}$ and complex spatial patterns ${ }^{51}$ is impaired by focal lesions in the associational occipitotemporal cortical areas that are known to be involved with the encoding of these stimulus features. Memoryguided saccades are less accurate in patients with damage to the occipito-parietal cortex and the $\mathrm{PFC}^{52,53}$, which are involved in the control of saccadic eye movements in monkeys. In the study shown in FIG. 5, 23 patients with surgical resections of temporal or parietal cortex performed simultaneous and delayed speed-discrimination tasks. Patients with damage to the left superior temporal cortex, and in particular the lateral superior temporal cortex (FIG. 5b), showed impaired speed discrimination as well as reduced memory for stimulus speed. The lateral superior temporal cortex in humans corresponds 

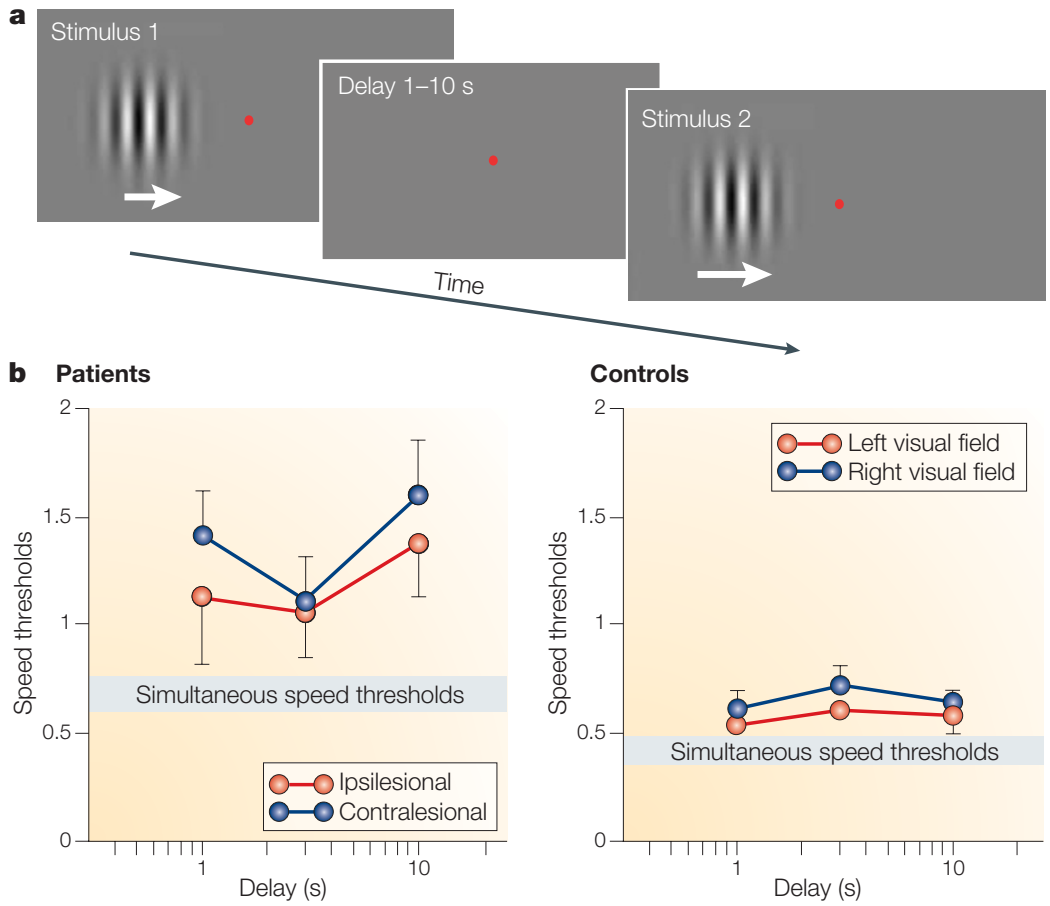

Figure 5 | Sensory working memory in patients with focal brain lesions. In this study ${ }^{48}$, the effects of brain lesions on the delayed discrimination of stimulus speed were assessed. a Experimental design used to assess speed memory. The sample and test stimuli moved in the same direction but could differ in speed, and were separated by a 1-10-s delay. $\mathbf{b} \mid$ Results of delayed discrimination tasks for patients with lesions in the superior temporal cortex (left), as compared with the results of age-matched controls (right). Grey shaded region denotes the mean value \pm 1 SEM of simultaneous (left versus right) speed discrimination thresholds. Although the patients showed slightly elevated thresholds in the simultaneous task, their thresholds rose sharply with increasing delay duration. The effect is more pronounced for stimuli presented in the visual field contralateral to the brain-damaged hemisphere.

DELAYED MATCHING-TOSAMPLE TASKS Presentation of a stimulus is followed by a delay, after which a choice is offered. In matching tasks, the original stimulus that was presented must be chosen; in non-matching tasks, a new stimulus must be selected. With small stimulus sets, the stimuli are frequently repeated and become highly familiar. So, such tasks are most readily solved by short-term or working memory rather than by long-term memory. In the original version of this task, trained animals or instructed volunteers report whether two stimuli separated by a memory delay are the same or different. approximately to area MST in the extrastriate cortex of macaque monkeys, and similar impairments have been reported in monkeys with damage to the motionsensitive MT/MST region ${ }^{54}$. These results support a role for extrastriate and associative visual cortex in the shortterm storage of velocity information.

Stimulation. Bisley et al. ${ }^{20}$ found that microstimulation of direction-specific cortical columns in area MT of monkeys that were performing a memory-for-visualmotion task affected performance during both encoding and retention. However, the nature of these effects depended on the phase of the task during which the stimulation was delivered (FIG. 6).

Stimulation applied during presentation of the sample caused performance to drop to significantly below chance levels. Further analysis revealed that on these trials the monkeys nearly always identified the direction of the sample as that preferred by the stimulated column, irrespective of the actual sample stimulus. So, stimulation during the sample produced signals that were interpreted by the monkeys as directional motion. Stimulation during the delay period also affected performance, but the signals it produced were disruptive, bringing performance to chance levels. The effects of microstimulation during visual stimulation showed that the directional information used by the monkeys in the task was provided by neurons in area MT. The disruptive effects of stimulation during the delay indicates that MT neurons also participate in the storage of visual motion information, either by being connected to the storage circuitry or by being an integral component of that circuitry.

TRANSCRANIAL MAGNETIC STIMULATION (TMS) has also been used to examine the contribution of cortical areas that process sensory information to working memory in humans. For example, when TMS was used to disrupt activity in human area MT, motion PRIMING was eliminated $^{55}$. As this effect was specific to visual motion and to area MT, the authors concluded that priming for basic stimulus attributes depends on the intermediate stages of cortical visual processing. A recent psychophysical study of priming ${ }^{56}$ confirmed that idea.

\section{Tactile working memory}

Psychophysics. Early studies showed that tactile information (for example, for three-dimensional shapes, location and duration of stimulation) can be preserved for up to $80 \mathrm{~s}$ even when intervening tasks prevent rehearsal ${ }^{57,58}$. In a more recent study, Sinclair and Burton $^{59}$ found that, although subjects could perform delayed discrimination of vibration stimuli with delays of many seconds, the accuracy of this discrimination was maximal at short delays and decreased rapidly during the first $5 \mathrm{~s}$ of the delay. During longer delays, performance did not continue to deteriorate, indicating that a two-stage memory process might be involved. Monkeys can also accurately retain information about vibratory stimuli for many seconds ${ }^{60}$.

These studies reveal that, like visual stimuli, the remembered tactile stimuli are faithfully represented in working memory. This indicates that the regions that encode tactile information might be needed for this representation to persist during the memory delay.

Physiology. The work of Romo and colleagues provided some of the most compelling evidence in support of the involvement of somatosensory cortical neurons in working memory tasks. They recorded neuronal activity in cortical areas SI and SII during a delayed discrimination task that involved mechanical vibration (flutter) applied to a monkey's fingertip ${ }^{61,62}$. The monkey had to judge which of two stimuli separated by a delay had the higher frequency. The authors found no delay activity in area SI (REF. 63). However, in area SII (the subsequent processing stage), about a third of neurons that were significantly modulated by stimulus frequency continued to fire for several hundred milliseconds into the 3-s delay. Furthermore, their responses to the second stimulus contained information about the remembered stimulus, reflecting the relationship between the two stimuli, which was the problem that the monkey was asked to solve ${ }^{64}$. Romo and his colleagues also recorded the activity of other cortical areas, including premotor and prefrontal regions, during the same task. These studies provided a unique insight into the circuitry that underlies the performance of a tactile working memory task (see below). 
a Stimulate during sample
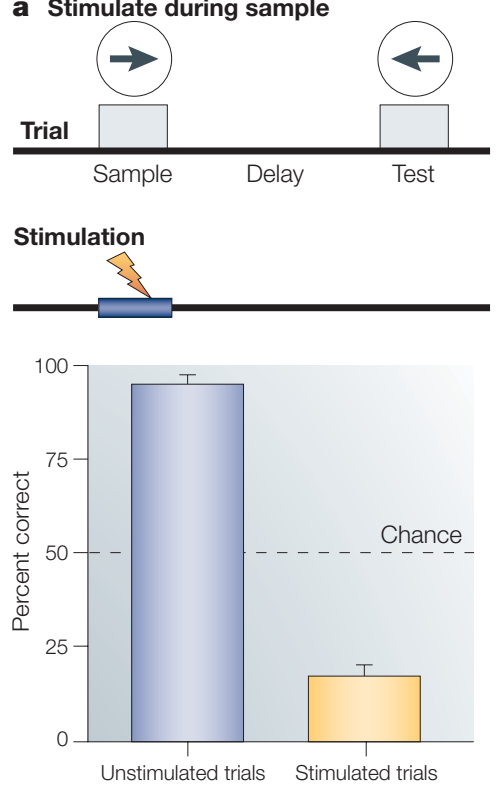

b Stimulate during delay

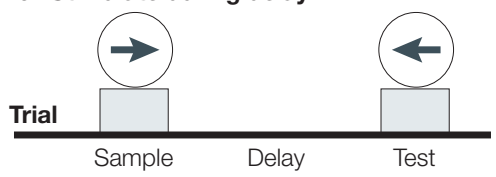

Stimulation
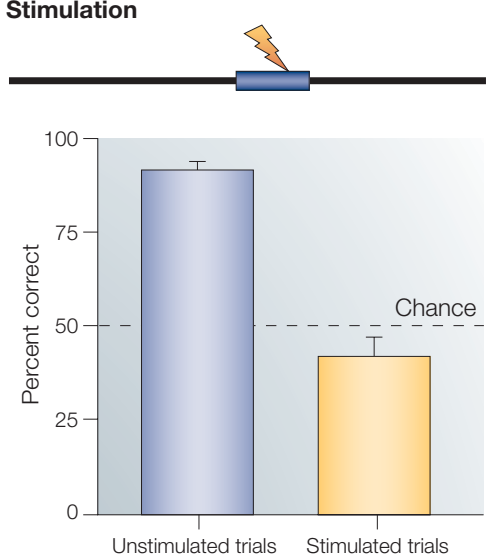

Figure 6 | Effect of electrical microstimulation of area MT on memory for motion. The timing of the sample, test and electrical microstimulation are shown diagrammatically (top). Microstimulation ( $80 \mathrm{~mA}$, biphasic $200 \mathrm{~Hz} ; 500$ ms duration) of identified directional columns in MT was applied on $25 \%$ of trials chosen at random and was either coincident with the presentation of the sample stimulus (a) or was applied during the delay (b). The two conditions were presented in separate blocks of trials. When microstimulation was applied during the sample, the performance dropped to $15 \%$ correct (a). Further analysis revealed that the animals consistently reported that the sample moved in the direction preferred by the stimulated cluster of neurons, showing that the signal produced by the microstimulation was interpreted as visual motion. When the same microstimulation was applied during the delay, it no longer produced consistent directional signals; the performance dropped to near chance. These effects indicate that MT neurons participate in storage of motion information by either maintaining an active connection with the circuitry involved in storage or by being an integral component of that circuitry. Modified, with permission, from REF. 20 @ (2001) American Physiological Society.

TRANSCRANIAL MAGNETIC STIMULATION

A technique that is used to induce a transient interruption of normal activity in a relatively restricted area of the brain. It is based on the generation of a strong magnetic field near the area of interest, which, if changed rapidly enough, will induce an electric field that is sufficient to stimulate neurons.

PRIMING

The facilitation of recognition, reproduction or biases in the selection of stimuli that have recently been perceived.
Although these studies found no memory-related activity in area SI, experiments by Fuster and colleagues did. These studies reported stimulus-related delay activity in neurons in SI and in other regions that have been implicated in texture analysis in monkeys that were trained on a memory task involving texture discriminations $\mathrm{s}^{23,65}$.

Imaging. Several imaging studies have focused on the ability to recognize and remember objects on the basis of tactile input ${ }^{66}$. For example, positron emission tomography (PET) studies have shown activation in the parietal operculum (area SII), an associative somatosensory area, during working memory tasks that use vibratory stimuli ${ }^{67}$ or palpated wire forms ${ }^{68}$. As a rule, these activations were accompanied by increased activity in other cortical regions, including the dorsolateral PFC. Information gained by tactile palpation of objects can be stored in working memory and used for discriminations. In a sequential discrimination $\operatorname{task}^{69}$, functional magnetic resonance imaging (fMRI) showed delayrelated activity in the PFC, frontal operculum and anterior parietal cortex. This activity could reflect the maintenance of non-visual object information over the delay period. Interestingly, there is considerable overlap between the prefrontal activations for tasks that involve haptic object encoding and those that involve visual encoding. Perhaps three-dimensional object information is extracted and stored in a supramodal fashion to allow recognition based on information flowing in from the different senses. In this manner, working memory would assist early-level sensory processes to form mental representations of objects that are explored in more than one sensory domain.

Transcranial magnetic stimulation. TMS has also been used to investigate the neuronal basis of memory for vibration frequency. When subjects were asked to compare the frequencies of two vibrations that were separated by a 1.5-s delay, TMS applied to area SI on the contralateral side in the early phase of the retention period increased the threshold for discrimination, whereas TMS later in the delay did not ${ }^{70}$. The authors concluded that area SI processes and stores vibration information, directly contributing to working memory. Physiological recordings from areas SI and SII in monkeys support this idea (see above).

\section{Auditory working memory}

Psychophysics. Humans can accurately discriminate the pitch and loudness of sounds $\mathrm{s}^{71}$, and our auditory working memory can persist for many seconds ${ }^{72}$. Like experiments on visual working memory, auditory memory studies also use interfering stimuli during the memory delay to investigate the nature of remembered stimuli. For example, memory for pitch can be disturbed by distractor tones, but only if the tones are within a narrow range of frequencies relative to the frequency of the remembered stimulus, supporting the hypothesis that there are separate pitch memory modules with inhibitory interactions ${ }^{73,74}$. In an auditory memory task, discrimination thresholds for pitch and loudness decline at different rates with increasing delay ${ }^{75}$ - intensity discrimination decreases more rapidly than frequency discrimination, indicating that pitch and loudness are processed separately in auditory memory. The differences in the precision of memory for intensity and pitch are parallel to the findings in vision for contrast and spatial frequency (see above). Furthermore, there is evidence that sound frequency is stored separately from location informa$\operatorname{tion}^{76,77}$. These findings indicate that, like visual memory, auditory memory involves separate stores for important physical parameters of the remembered stimuli.

Physiology. To study neuronal activity in the auditory cortex, Gottlieb et al. ${ }^{78}$ trained a monkey to compare two pure tones separated by a brief memory delay. During the delay, the firing rates of many neurons reflected the frequency of the first tone. In addition, in a subset of neurons, responses to the second tone depended on whether its frequency matched that of the first. These results strongly implicate auditory cortex neurons in the circuitry that underlies working memory for tone frequency. The participation of the auditory cortex in auditory working memory is also supported by a MAGNETOENCEPHALOGRAPHY study that investigated memory for tone loudness ${ }^{79}$. In addition, the auditory 


\section{Box 2 | Computational models of working memory}

Interactions between the prefrontal cortex (PFC) and sensory areas have been incorporated into biophysically based models that are designed to account for persistent activity in the $\mathrm{PFC}^{95,108}$. In one class of models, a working memory network is characterized by multiple attractor states, each of which retains a representation of a stimulus to be remembered ${ }^{109}$. Sensory information is fed into a network of neurons that are involved in parametric storage of analogue stimulus dimensions such as spatial location or tactile vibration, and this storage is mediated by a neural assembly that has a resting state at low firing rates and a self-sustaining 'attractor' state at elevated firing rates. Such 'attractor' models have provided a realistic account of several types of delay activity that have been recorded in the $\mathrm{PFC}^{110,111}$ and inferotemporal cortex ${ }^{109}$. One of the features of such recurrent networks is that they are likely to be disrupted by distractors that resemble the stimulus retained by such networks ${ }^{110}$. This behaviour is reminiscent of the 'memory masking' that is observed in psychophysical experiments (FIG. 2).

MAGNETOENCEPHALOGRAPHY

A non-invasive technique that allows the detection of the changing magnetic fields that are associated with brain activity. As the magnetic fields of the brain are weak, extremely sensitive magnetic detectors known as superconducting quantum interference devices — which work at very low, superconducting temperatures $\left(-269^{\circ} \mathrm{C}\right)$ - are used to pick up the signal. cortex is connected to regions in the $\mathrm{PFC}^{80}$ that have been implicated in working memory (BOX 1), which further supports the involvement of this key sensory region in the retention of auditory stimuli.

Imaging. Several imaging studies have focused on the ability to remember differences in pitch and tonal sequences. In these studies, tonal sequences produced memory-related activity in associative auditory cortex in the right superior temporal lobe, and pitch judgements were associated with increased activation in the right frontal lobe ${ }^{81,82}$. There is also evidence that the activation in the auditory cortex is greatest early in the delay, whereas regions in the supramarginal gyrus and parts of the cerebellum are activated later, indicating that these areas have different roles in memory for pitch $^{83}$.

Lesions. Patients with damage to auditory associative cortex in the right temporal lobe show selective deficits in short-term storage of auditory information. These patients are unimpaired on a tone discrimination task with a short (1.65-s) delay, but show deficits when a longer delay with an interfering tone sequence is introduced ${ }^{84}$.

\section{Conclusions}

We have reviewed the evidence that the circuitry that underlies working memory for sensory signals is likely to include cortical areas that are devoted to the processing of these signals. This conclusion is supported by the results of a wide range of studies, from purely perceptual measurements to recordings from single cells and functional imaging. Manipulations such as lesions and stimulation provide further confirmation of this hypothesis, which is also supported by computational modelling (BOX 2).
One of the difficulties we encountered while reviewing this evidence was the difference in the treatment and analysis of sensory stimuli used in memory tasks. Studies that use exclusively psychophysical approaches tend to focus on the precise nature of the sensory representation, carefully manipulating stimuli used in memory tasks along a well-defined sensory dimension. For example, visual psychophysical studies indicate that information about stimulus size (spatial frequency) is likely to be preserved by distinct, narrowly tuned mechanisms, and that information about the motion of random dots is preserved with information about its precise speed and location. Similarly, investigations of auditory working memory have provided evidence that information about pitch, loudness and location is preserved by separate, narrowly tuned mechanisms.

On the other hand, single-cell recording studies have focused largely on the nature of memory-related neural activity and its localization in the cortex. Although such studies have provided invaluable insights into the nature of neural activity during the performance of working memory tasks, few have shed light on how this activity represents the remembered sensory stimuli. Among studies that have focused on the nature of sensory representation are those by Romo and colleagues ${ }^{61-64}$. By parametrically manipulating a vibratory stimulus they showed that, during the memory period, information about vibration frequency was preserved not only in area SII but also in the PFC and the premotor cortical area, shedding light on the circuitry that underlies working memory in this task.

The main strength of brain imaging studies is their ability to reveal simultaneous activation of multiple cortical regions during working memory tasks. These studies lend further support to the role of mid-level sensory areas that interact with the PFC and the parietal cortex during these tasks. However, with few exceptions, these studies have mainly produced evidence for a coarse subdivision of 'what' and 'where' aspects of information processing. Like single-cell recordings, imaging studies of sensory working memory would benefit from attempts to focus on more specific aspects of the stimulus material being remembered, to support or reject the theory of feature-specific memory stores.

Although the participation of sensory cortical areas in the circuitry that temporarily stores sensory information is well documented, the precise nature of this participation is still unclear. We believe that to understand how the brain deals with this important problem, we must devote more attention to exactly what is remembered.
1. Baddeley, A. Working Memory (Oxford Univ. Press, Oxford, 1986).

2. Regan, D. Storage of spatial-frequency information and spatial frequency discrimination. J. Opt. Soc. Am. A. 2 , 619-621 (1985).

3. Pasternak, T., Bisley, J. W. \& Calkins, D. in Biological Psychology (eds Gallagher, M. \& Nelson, R. J.) 130-185 (John Wiley \& Sons Inc. New York, 2003). A comprehensive review of the organization of and processing in the visual system of primates.

4. Magnussen, S. Low-level memory processes in vision. Trends Neurosci. 23, 247-251 (2000).
5. Magnussen, S., Greenlee, M. W., Asplund, R. \& Dyrnes, S. Stimulus specific mechanisms of visual short-term memory. Vision Res. 31, 1213-1219 (1991).

6. Magnussen, S. \& Greenlee, M. W. Retention and disruption of motion information in visual short-term memory. J. Exp. Psychol. Learn. Mem. Cogn. 18, 151-156 (1992). This study introduces a memory masking paradigm, in which an interfering distractor stimulus is presented during the memory period of a delayed match-to-sample task.

7. Magnussen, S., Greenlee, M. W. \& Thomas, J. P. Parallel processing in visual short-term memory.
J. Exp. Psychol. Hum. Percept. Perform. 22, 202-212 (1996)

8. Mclntosh, A. R. et al. Recruitment of unique neural systems to support visual memory in normal aging. Curr. Biol. $\mathbf{9}$, 1275-1278 (1999).

9. Magnussen, S., Idas, E. \& Myhre, S. H. Representation of orientation and spatial frequency in perception and memory: a choice reaction-time analysis. J. Exp. Psychol. Hum. Percept. Perform. 24, 707-718 (1998).

10. Vogels, R. \& Orban, G. A. Decision processes in visual discrimination of line orientation. J. Exp. Psychol. Hum. Percept. Perform. 12, 115-132 (1986). 
11. Korsnes, M. S. \& Magnussen, S. Age comparisons of serial position effects in short-term memory. Acta Psychol. (Amst.) 94, 133-143 (1996).

12. Lee, B. \& Harris, J. Contrast transfer characteristics of visual short-term memory. Vision Res. 36, 2159-2166 (1996).

13. Harvey, L. O. in Human Memory and Cognitive Capabilities (eds Clix, F. \& Hagendorf, H.) 173-187 (Elsevier, Amsterdam, 1986).

14. Fahle, M. \& Harris, J. P. Visual memory for vernier offsets. Vision Res. 32, 1033-1042 (1992)

15. Bisley, J. W. \& Pasternak, T. The multiple roles of visual cortical areas MT/MST in remembering the direction of visual motion. Cereb. Cortex 10, 1053-1065 (2000). A lesion study showing that the effects of MT/MST lesions on memory for motion depends on the nature of the stimuli and of the task. With complex motion stimuli, the lesion affected both processing and retention of directional information. However, with coherently moving stimuli, the lesions affected only the comparison component of the task without affecting working memory.

16. Magnussen, S. \& Greenlee, M. W. The psychophysics of perceptual memory. Psychol. Res. 62, 81-92 (1999).

17. Lalonde, J. \& Chaudhuri, A. Task-dependent transfer of perceptual to memory representations during delayed spatial frequency discrimination. Vision Res. 42, 1759-1769 (2002).

19. Blake, R., Cepeda, N. J. \& Hiris, E. Memory for visual motion. J. Exp. Psychol. Hum. Percept. Perform. 23, 353-369 (1997).

20. Bisley, J. W., Zaksas, D. \& Pasternak, T. Microstimulation of cortical area MT affects performance on a visual working memory task. J. Neurophysiol. 85, 187-196 (2001). Electrical stimulation of area MT during the presentation of the sample caused monkeys to report motion in the direction preferred by the stimulated neurons. The same stimulation delivered during the memory delay disrupted performance but did not produce such a bias. This result indicates that MT is part of, or is connected to, the circuitry that underlies working memory for motion.

21. Zaksas, D., Bisley, J. W. \& Pasternak, T. Motion information is spatially localized in a visual working-memory task. J. Neurophysiol. 86, 912-921 (2001).

22. Kahana, M. J. \& Sekuler, R. Recognizing spatial patterns: a noisy exemplar approach. Vision Res. 42, 2177-2192 (2002)

23. Zhou, Y. D. \& Fuster, J. M. Mnemonic neuronal activity in somatosensory cortex. Proc. Natl Acad. Sci. USA 93, 10533-10537 (1996)

24. Gegenfurtner, K. Cortical mechanisms of colour vision Nature Rev. Neurosci. 4, 563-572 (2003).

25. Nilsson, T. H. \& Nelson, T. M. Delayed monochromatic hue matches indicate characteristics of visual memory. J. Exp. Psychol. Hum. Percept. Perform. 7, 141-150 (1981).

26. Vuontela, V., Rama, P., Raninen, A., Aronen, H. J. \& Carlson, S. Selective interference reveals dissociation between memory for location and colour. Neuroreport 10 2235-2240 (1999).

27. Ungerleider, L. \& Pasternak, T. in Visual Neurosciences (eds Chalupa, L. M. \& Werner, J. S.) 541-563 (MIT Press, Cambridge, Massachusetts, 2004).

28. Fuster, J. M. Inferotemporal units in selective visual attention and short-term memory. J. Neurophysiol. 64, 681-697 (1990).

29. Miller, E. K., Li, L. \& Desimone, R. Activity of neurons in anterior inferior temporal cortex during a short-term memory task. J. Neurophysiol. 13, 1460-1478 (1993).

30. Miyashita, Y. \& Chang, H. S. Neuronal correlate of pictorial short-term memory in the primate temporal cortex. Nature 331, 68-70 (1988). A study showing that visual working memory is reflected in the sustained activity of individual IT neurons.

31. Chelazzi, L., Duncan, J., Miller, E. K. \& Desimone, R. Responses of neurons in inferior temporal cortex during memory-guided visual search. J. Neurophysiol. $\mathbf{8 0}$, 2918-2940 (1998)

32. Ferrera, V. P., Rudolph, K. K. \& Maunsell, J. H. Responses of neurons in the parietal and temporal visual pathways during a motion task. J. Neurophysiol. 14, 6171-6186 (1994).

33. Motter, B. C. Neural correlates of feature selective memory and pop-out in extrastriate area V4. J. Neurosci. 14, 2190-2199 (1994).

34. Constantinidis, C. \& Steinmetz, M. A. Neuronal activity in posterior parietal area 7 a during the delay periods of a spatial memory task. J. Neurophysiol. 76, 1352-1355 (1996)

35. Bisley, J. W., Zaksas, D., Droll, J. \& Pasternak, T. Activity of neurons in cortical area MT during a memory for motion task. J. Neurophysiol. 91, 286-300 (2004).
A recording study showing that MT neurons are active not only during the presentation of visual motion stimuli but also during the memory delay. This activity carries signals that reflect the direction of the remembered stimulus.

36. Courtney, S. M., Petit, L., Maisog, J. M., Ungerleider, L. G. \& Haxby, J. V. An area specialized for spatial working memory in human frontal cortex. Science 279, 1347-1351 (1998).

37. Smith, E. E. \& Jonides, J. Storage and executive processes in the frontal lobes. Science 283, 1657-1661 (1999).

38. Greenlee, M. W., Magnussen, S. \& Reinvang, I. Brain regions involved in spatial frequency discrimination: evidence from fMRI. Exp. Brain Res. 132, 399-403 (2000). A neuroimaging study that used a delayed match-tosample task to investigate the retention of spatial frequency information. The memory task produced elevated activity in the prefrontal, parietal and occipital cortex.

39. Pessoa, L., Gutierrez, E., Bandettini, P. \& Ungerleider, L. Neural correlates of visual working memory: fMRI amplitude predicts task performance. Neuron 35, 975-987 (2002) An event-related neuroimaging study in which the subject had to detect a small orientation change in a single element of a line-element texture pattern. Trials with correct responses were associated with elevated activity in the prefrontal and parietal cortex, whereas those with incorrect responses were not

40. Courtney, S. M., Ungerleider, L. G., Keil, K. \& Haxby, J. V. Transient and sustained activity in a distributed neural system for human working memory. Nature 386, 608-611 (1997).

41. Cornette, L., Dupont, P., Salmon, E. \& Orban, G. A. The neural substrate of orientation working memory. J. Cogn. Neurosci. 13, 813-828 (2001).

42. McCarthy, G. et al. Brain activation associated with visual motion studied by functional magnetic resonance imaging in humans. Hum. Brain Mapp. 2, 234-243 (1994).

43. Fuster, J. M., Bauer, R. H. \& Jervey, J. P. Effects of cooling inferotemporal cortex on performance of visual memory tasks. Exp. Neurol. 71, 398-409 (1981).

44. Fuster, J. M., Bauer, R. H. \& Jervey, J. P. Functiona interactions between inferotemporal and prefrontal cortex in a cognitive task. Brain Res. 330, 299-307 (1985).

45. Frey, S. \& Petrides, M. Orbitofrontal cortex: a key prefronta region for encoding information. Proc. Natl Acad. Sci. USA 97, 8723-8727 (2000).

46. Walsh, V., Le Mare, C., Blaimire, A. \& Cowey, A. Normal discrimination performance accompanied by priming deficits in monkeys with V4 or TEO lesions. Neuroreport 11, 1459-1462 (2000).

47. Greenlee, M. W., Rischewski, J., Mergner, T. \& Seeger, W. Delayed pattern discrimination in patients with unilateral temporal lobe damage. J. Neurosci. 13, 2565-2574 (1993).

48. Greenlee, M. W. Lang, H.-J., Mergner, T. \& Seeger, W. Visual short-term memory of stimulus velocity in patients with unilateral posterior brain damage. J. Neurosci. 15, 2287-2300 (1995).

A visual working memory study in a group of patients with brain damage, who had to discriminate the relative speeds of moving gratings. The results indicate that the damaged extrastriate area MT+ is associated with a decline in discrimination performance that was more pronounced at long delays.

49. Davidoff, J. B. \& Ostergaard, A. L. Colour anomia resulting from weakened short-term colour memory. A case study. Brain 107, 415-431 (1984).

50. Schoppig, A. et al. Short-term memory for colour following posterior hemispheric lesions in man. Neuroreport 10 1379-1384 (1999).

51. Greenlee, M. W., Koessler, M., Cornelissen, F. W. \& Mergner, T. Visual discrimination and short-term memory for random patterns in patients with a focal cortical lesion. Cereb. Cortex 7, 253-267 (1997).

52. Pierrot-Deseilligny, C., Rivaud, S., Penet, C. \& Rigolet, M. H. Latencies of visually guided saccades in unilateral hemispheric cerebral lesions. Ann. Neurol. 21, 138-148 (1987).

53. Greenlee, M. W., Berg, H., Stuhr, V. \& Mergner, T. Visual search and visual working memory in patients with chronic focal cortical lesions. Vision Res. 40, 3759-3773 (2000).

54. Merigan, W. H. \& Pasternak, T. in The Neuropsychology of Vision (eds Fahle, M. \& Greenlee, M.) 121-162 (Oxford Univ. Press, Oxford, 2003)

55. Campana, G., Cowey, A. \& Walsh, V. Priming of motion direction and area V5/MT: a test of perceptual memory. Cereb. Cortex 12, 663-669 (2002).

56. Bar, M. \& Biederman, I. Localizing the cortical region mediating visual awareness of object identity. Proc. Natl Acad. Sci. USA 96, 1790-1793 (1999).
57. Gilson, E. Q. \& Baddeley, A. Tactile short-term memon Q. J. Exp. Psychol. 21, 180-184 (1969).

58. Sullivan, E. V. \& Turvey, M. Short-term retention of tactile stimulation. Q. J. Exp. Psychol. 24, 253-261 (1972).

59. Sinclair, R. J. \& Burton, H. Discrimination of vibrotactile frequencies in a delayed pair comparison task. Percept. Psychophys. 58, 680-692 (1996).

60. Hernandez, A., Salinas, E., Garcia, R. \& Romo, R. Discrimination in the sense of flutter: new psychophysical measurements in monkeys. J. Neurosci. 17, 6391-6400 (1997).

61. Hernandez, A., Zainos, A. \& Romo, R. Neuronal correlates of sensory discrimination in the somatosensory cortex. Proc. Natl Acad. Sci. USA 97, 6191-6196 (2000).

62. Romo, R. \& Salinas, E. Touch and go: decision-making mechanisms in somatosensation. Annu. Rev. Neurosci. 24 107-137 (2001)

63. Romo, R. \& Salinas, E. Flutter discrimination: neural codes, perception, memory and decision making. Nature Rev. Neurosci. 4, 203-218 (2003).

A review of elegant experiments in which Romo and Salinas combined psychophysical and neurophysiological approaches to examine the cortical mechanisms involved in the performance of a vibrotactile delayed discrimination task.

64. Romo, R., Hernandez, A., Zainos, A., Lemus, L. \& Brody, C. D. Neuronal correlates of decision-making in secondary somatosensory cortex. Nature Neurosci. 5, 1217-1225 (2002)

65. Koch, K. W. \& Fuster, J. M. Unit activity in monkey parietal cortex related to haptic perception and temporary memory. Exp. Brain Res. 76, 292-306 (1989).

66. Burton, H. \& Sinclair, R. J. Attending to and remembering tactile stimuli: a review of brain imaging data and singleneuron responses. J. Clin. Neurophysiol. 17, 575-591 (2000).

67. Klingberg, T., Kawashima, R. \& Roland, P. E. Activation of multi-modal cortical areas underlies short-term memory. Eur. J. Neurosci. 8, 1965-1971 (1996).

68. Bonda, E., Petrides, M. \& Evans, A. Neural systems for tactual memories. J. Neurophysiol. 75, 1730-1737 (1996).

69. Stoeckel, M. C. et al. A fronto-parietal circuit for tactile object discrimination: an event-related fMRI study. Neuroimage 19, 1103-1114 (2003).

70. Harris, J. A., Miniussi, C., Harris, I. M. \& Diamond, M. E. Transient storage of a tactile memory trace in primary somatosensory cortex. J. Neurosci. 22, 8720-8725 (2002). This study reported the effects of TMS applied over the somatosensory cortex (area SI) on the ability to discriminate and remember the frequency of vibration (flutter). TMS pulses delivered to area SI on the contralateral side early in the delay period disrupted memory performance, whereas the same pulses delivered late in the delay or to the ipsilateral area SI had no effect.

71. Posner, M. I. Short term memory systems in human information processing. Acta Psychol. (Amst.) 27, 267-284 (1967).

72. Samms, M., Hari, R., Rif, J. \& Knuutila, J. The human auditor sensory memory trace persists about 10 sec: neuromagnetic evidence. J. Cogn. Neurosci. 5, 363-370 (1993).

73. Deutsch, D. Mapping of interactions in the pitch memory store. Science 175, 1020-1022 (1972).

74. Deutsch, D. Interference in memory between tones adjacent in the musical scale. J. Exp. Psychol. 100, 228-231 (1973).

75. Clement, S., Demany, L. \& Semal, C. Memory for pitch versus memory for loudness. J. Acoust. Soc. Am. 106 2805-2811 (1999).

76. Anourova, I. et al. Selective interference reveals dissociation between auditory memory for location and pitch. Neuroreport 10, 3543-3547 (1999).

77. Clarke, S., Adriani, M. \& Bellmann, A. Distinct short-term memory systems for sound content and sound localization. Neuroreport 9, 3433-3437 (1998)

78. Gottlieb, Y., Vaadia, E. \& Abeles, M. Single unit activity in the auditory cortex of a monkey performing a short term memory task. Exp. Brain Res. 74, 139-148 (1989). This study showed that during the performance of a delayed discrimination task involving tone frequencies, the activity of neurons in the primary auditory cortex of monkeys carried signals about the remembered tone frequency. These results implicate neurons in auditory cortex in the circuitry that underlies auditory working memory.

79. Lu, Z. L., Williamson, S. J. \& Kaufman, L. Behavioral lifetime of human auditory sensory memory predicted by physiological measures. Science 258, 1668-1670 (1992).

80. Romanski, L. M., Bates, J. F. \& Goldman-Rakic, P. S. Auditory belt and parabelt projections to the prefrontal cortex in the rhesus monkey. J. Comp. Neurol. $\mathbf{4 0 3 ,}$ 141-157 (1999). 
81. Zatorre, R. J., Evans, A. C. \& Meyer, E. Neural mechanisms underlying melodic perception and memory for pitch. J. Neurosci. 14, 1908-1919 (1994).

82. Gaab, N., Gaser, C., Zaehle, T., Jancke, L. \& Schlaug, G. Functional anatomy of pitch memory - an fMRI study with sparse temporal sampling. Neuroimage 19, 1417-1426 (2003).

83. Mathiak, K., Hertrich, I., Grodd, W. \& Ackermann, H. Discrimination of temporal information at the cerebellum: functional magnetic resonance imaging of nonverbal auditory memory. Neuroimage 21, 154-162 (2004).

84. Zatorre, R. J. \& Samson, S. Role of the right temporal neocortex in retention of pitch in auditory short-term memory. Brain 114, 2403-2417 (1991).

85. Levy, R. \& Goldman-Rakic, P. S. Segregation of working memory functions within the dorsolateral prefrontal cortex. Exp. Brain Res. 133, 23-32 (2000).

This review describes the evidence in support of a modular 'domain-specific' organization of the PFC and the role of this organization in sensory storage.

86. Barbas, H. Anatomic organization of basoventral and mediodorsal visual recipient prefrontal regions in the rhesus monkey. J. Comp. Neurol. 276, 313-342 (1988).

87. Carmichael, S. T. \& Price, J. L. Sensory and premotor connections of the orbital and medial prefrontal cortex of macaque monkeys. J. Comp. Neurol. 363, 642-664 (1995).

88. Romo, R., Brody, C. D., Hernandez, A. \& Lemus, L. Neuronal correlates of parametric working memory in the prefrontal cortex. Nature 399, 470-473 (1999).

89. Romanski, L. M. \& Goldman-Rakic, P. S. An auditory domain in primate prefrontal cortex. Nature Neurosci. $\mathbf{5}$ 15-16 (2002).

90. Funahashi, S., Bruce, C. J. \& Goldman-Rakic, P. S. Visuospatial coding in primate prefrontal neurons revealed by oculomotor paradigms. J. Neurophysiol. 63, 814-831 (1990).

91. Fuster, J. M., Bodner, M. \& Kroger, J. K. Cross-modal and cross-temporal association in neurons of frontal cortex. Nature 405, 347-351 (2000)

92. Bodner, M., Kroger, J.\& Fuster, J. M. Auditory memory cells in dorsolateral prefrontal cortex. Neuroreport 7, 1905-1908 (1996).

93. Rao, S. C., Rainer, G. \& Miller, E. K. Integration of what and where in the primate prefrontal cortex. Science $\mathbf{2 7 6}$ 821-824 (1997)
94. Barbas, H. \& Pandya, D. N. Architecture and intrinsic connections of the prefrontal cortex in the rhesus monkey. J. Comp. Neurol. 286, 353-375 (1989).

95. Wang, X. J. Synaptic reverberation underlying mnemonic persistent activity. Trends Neurosci. 24, 455-463 (2001).

96. Goldman-Rakic, P. S. Cellular basis of working memory Neuron 14, 477-485 (1995)

97. Miller, E. K., Erickson, C. A. \& Desimone, R. Neural mechanisms of visual working memory in prefrontal cortex of the macaque. J. Neurosci. 16, 5154-5167 (1996).

98. Fuster, J. M. Memory in the Cerebral Cortex: An Empirical Approach to Neural Networks in the Human and Nonhuman Primate (MIT Press, Cambridge, Massachusetts, 1999).

99. Rainer, G., Asaad, W. F. \& Miller, E. K. Selective representation of relevant information by neurons in the primate prefrontal cortex. Nature 393, 577-579 (1998).

100. Funahashi, S., Bruce, C. J. \& Goldman-Rakic, P. S. Mnemonic coding of visual space in the monkey's dorsolateral prefrontal cortex. J. Neurophysiol. 61, 331-349 (1989).

101. Constantinidis, C., Franowicz, M. N. \& Goldman-Rakic, P. S. The sensory nature of mnemonic representation in the primate prefrontal cortex. Nature Neurosci. 4, 311-316 (2001).

102. Fuster, J. Memory In The Cerebral Cortex (MIT Press, Cambridge, Massachusetts, 1995).

A unique and comprehensive overview of studies and theories exploring the neural basis of memory. Fuster, a pioneering memory researcher, presents a theory that short-term and working memory represent an updated temporary activation of cortical networks of long-term memory.

103. Bullier, J. in Visual Neurosciences (eds Chalupa, L. M. \& Werner, J. S.) 522-540 (MIT Press, Cambridge, Massachusetts, 2004)

104. Miller, E. K. \& Cohen, J. D. An integrative theory of prefrontal cortex function. Annu. Rev. Neurosci. 24, 167-202 (2001).

A comprehensive review of neurophysiological, neurobiological, neuroimaging and computational studies that support the theory that the PFC is responsible for executive control of other brain regions and pathways involved in behaviour.
105. Chafee, M. V. \& Goldman-Rakic, P. S. Inactivation of parietal and prefrontal cortex reveals interdependence of neural activity during memory-guided saccades. J. Neurophysiol. 83, 1550-1566 (2000).

106. Tomita, H., Ohbayashi, M., Nakahara, K., Hasegawa, I. \& Miyashita, Y. Top-down signal from prefrontal cortex in executive control of memory retrieval. Nature 401, 699-703 (1999).

107. Miller, E. K. The prefrontal cortex: complex neural properties for complex behavior. Neuron 22, 15-17 (1999).

108. Durstewitz, D., Seamans, J. K. \& Sejnowski, T. J. Neurocomputational models of working memory. Nature Neurosci. 3, 1184-1191 (2000)

109. Amit, D. J., Fusi, S. \& Yakovlev, V. Paradigmatic working memory (attractor) cell in IT cortex. Neural Comput. 9, 1071-1092 (1997).

110. Compte, A., Brunel, N., Goldman-Rakic, P. S. \& Wang, X. J. Synaptic mechanisms and network dynamics underlying spatial working memory in a cortical network model. Cereb. Cortex 10, 910-923 (2000).

111. Miller, P., Brody, C. D., Romo, R. \& Wang, X.-J. A recurrent network model of somatosensory parametric working memory in the prefrontal cortex. Cereb. Cortex 13, 1208-1218 (2003).

Acknowledgements

T. Pasternak was supported by a grant from the National Eye Institute and, in part, a grant to the Center for Visual Science. M. Greenlee acknowledges financial support from the German Research Foundation. We thank Gregor Rainer for comments on the early version of the manuscript.

Competing interests statement

The authors declare no competing financial interests.

\section{0) Online links}

\section{FURTHER INFORMATION}

Pasternak's homepage:

http://www.urmc.rochester.edu/gebs/faculty/tatiana_pasternak. htm

Greenlee's homepage: http://www.psychologie.uni-

regensburg.de/Greenlee/index1.html

Access to this interactive links box is free online. 